Research Article

\title{
On Alzer and Qiu's Conjecture for Complete Elliptic Integral and Inverse Hyperbolic Tangent Function
}

\author{
Yu-Ming Chu, Miao-Kun Wang, and Ye-Fang Qiu \\ Department of Mathematics, Huzhou Teachers College, Huzhou 313000, China \\ Correspondence should be addressed to Yu-Ming Chu, chuyuming2005@yahoo.com.cn \\ Received 23 May 2011; Accepted 16 August 2011 \\ Academic Editor: Dirk Aeyels
}

Copyright (C) 2011 Yu-Ming Chu et al. This is an open access article distributed under the Creative Commons Attribution License, which permits unrestricted use, distribution, and reproduction in any medium, provided the original work is properly cited.

We prove that the double inequality $(\pi / 2)(\operatorname{arth} r / r)^{3 / 4+\alpha^{*} r}<\mathcal{K}(r)<(\pi / 2)(\operatorname{arth} r / r)^{3 / 4+\beta^{*} r}$ holds for all $r \in(0,1)$ with the best possible constants $\alpha^{*}=0$ and $\beta^{*}=1 / 4$, which answer to an open problem proposed by Alzer and Qiu. Here, $\mathcal{K}(r)$ is the complete elliptic integrals of the first kind, and arth is the inverse hyperbolic tangent function.

\section{Introduction}

For $r \in[0,1]$, Lengedre's complete elliptic integrals of the first and second kind [1] are defined by

$$
\begin{gathered}
\mathcal{K}=\mathcal{K}(r)=\int_{0}^{\pi / 2}\left(1-r^{2} \sin ^{2} \theta\right)^{-1 / 2} d \theta, \\
\mathcal{K}^{\prime}=\mathcal{K}^{\prime}(r)=\mathcal{K}\left(r^{\prime}\right), \\
\mathcal{K}(0)=\frac{\pi}{2}, \quad \mathcal{K}(1)=\infty, \\
\mathcal{E}=\mathcal{E}(r)=\int_{0}^{\pi / 2}\left(1-r^{2} \sin ^{2} \theta\right)^{1 / 2} d \theta, \\
\mathcal{E}^{\prime}=\mathcal{E}^{\prime}(r)=\mathcal{E}\left(r^{\prime}\right), \\
\mathcal{E}(0)=\frac{\pi}{2}, \quad \mathcal{E}(1)=1,
\end{gathered}
$$


respectively. Here and in what follows, we set $r^{\prime}=\sqrt{1-r^{2}}$. These integrals are special cases of Guassian hypergeometric function

$$
F_{2}(a, b ; c ; x)=F_{1}(a, b ; c ; x)=\sum_{n=0}^{\infty} \frac{(a, n)(b, n)}{(c, n)} \frac{x^{n}}{n !} \quad(-1<x<1),
$$

where $(a, n)=\prod_{k=0}^{n-1}(a+k)$. Indeed, we have

$$
\mathcal{K}(r)=\frac{\pi}{2} F\left(\frac{1}{2}, \frac{1}{2} ; 1 ; r^{2}\right), \quad \mathcal{E}(r)=\frac{\pi}{2} F\left(-\frac{1}{2}, \frac{1}{2} ; 1 ; r^{2}\right) .
$$

It is well known that the complete elliptic integrals have many important applications in physics, engineering, geometric function theory, quasiconformal analysis, theory of mean values, number theory, and other related fields [2-13].

Recently, the complete elliptic integrals have been the subject of intensive research. In particular, many remarkable properties and inequalities can be found in the literature [3, 1018].

In 1992, Anderson et al. [15] discovered that $\mathcal{K}$ can be approximated by the inverse hyperbolic tangent function, arth, and proved that

$$
\frac{\pi}{2}\left(\frac{\operatorname{arth} r}{r}\right)^{1 / 2}<\mathcal{K}(r)<\frac{\pi}{2}\left(\frac{\operatorname{arth} r}{r}\right),
$$

for $r \in(0,1)$.

In [16], Alzer and Qiu proved that the double inequality

$$
\frac{\pi}{2}\left(\frac{\operatorname{arth} r}{r}\right)^{\alpha}<\mathcal{K}(r)<\frac{\pi}{2}\left(\frac{\operatorname{arth} r}{r}\right)^{\beta}
$$

holds for all $r \in(0,1)$ with the best possible constants $\alpha=3 / 4$ and $\beta=1$ and proposed an open problem as follows.

\section{Open Problem \#}

The double inequality

$$
\frac{\pi}{2}\left(\frac{\operatorname{arth} r}{r}\right)^{3 / 4+\alpha^{*} r}<\mathcal{K}(r)<\frac{\pi}{2}\left(\frac{\operatorname{arth} r}{r}\right)^{3 / 4+\beta^{*} r},
$$

holds for all $r \in(0,1)$ with the best possible constants $\alpha^{*}=0$ and $\beta^{*}=1 / 4$.

It is the aim of this paper to give a positive answer to the open problem \#.

\section{Lemmas and Theorem}

In order to establish our main result, we need several formulas and lemmas, which we present in this section. 
For $0<r<1$, the following derivative formulas were presented in [4, Appendix E, pages 474-475]:

$$
\begin{array}{cl}
\frac{d \mathcal{K}}{d r}=\frac{\varepsilon-r^{\prime 2} \mathcal{K}}{r r^{\prime 2}}, & \frac{d \varepsilon}{d r}=\frac{\varepsilon-\mathcal{K}}{r}, \\
\frac{d\left(\mathcal{E}-r^{\prime 2} \mathcal{K}\right)}{d r}=r \mathcal{K}, & \frac{d(\mathcal{K}-\varepsilon)}{d r}=\frac{r \varepsilon}{r^{\prime 2}} .
\end{array}
$$

Lemma 2.1 (see [4, Theorem 1.25]). For $-\infty<a<b<\infty$, let $f, g:[a, b] \rightarrow \mathbb{R}$ be continuous on $[a, b]$ and be differentiable on $(a, b)$, let $g^{\prime}(x) \neq 0$ be on $(a, b)$. If $f^{\prime}(x) / g^{\prime}(x)$ is increasing (decreasing) on $(a, b)$, then so are

$$
\frac{f(x)-f(a)}{g(x)-g(a)}, \quad \frac{f(x)-f(b)}{g(x)-g(b)}
$$

If $f^{\prime}(x) / g^{\prime}(x)$ is strictly monotone, then the monotonicity in the conclusion is also strict.

The following Lemma 2.2 can be found in [9, Lemma 3(1)] and [4, Theorem 3.21(1) and Exercise 3.43(30) and (46)].

Lemma 2.2. (1) $\left[\left(r^{\prime}\right)^{c}\right.$ arth $\left.r\right] / r$ is strictly decreasing in $(0,1)$ if and only if $c \geq 2 / 3$;

(2) $\left(\varepsilon-r^{\prime 2} \mathcal{K}\right) / r^{2}$ is strictly increasing from $(0,1)$ onto $(\pi / 4,1)$;

(3) $\left(\varepsilon-r^{\prime 2} \mathcal{K}\right) /\left(r^{2} \mathcal{K}\right)$ is strictly decreasing from $(0,1)$ onto $(0,1 / 2)$;

(4) $r \mathcal{K} /$ arth $r$ is strictly decreasing from $(0,1)$ onto $(1, \pi / 2)$.

Lemma 2.3. (1) $f_{1}(r)=\left[r-r^{\prime 2}\right.$ arth $\left.r\right] / r^{3}$ is strictly increasing from $(0,1)$ onto $(2 / 3,1)$;

(2) $f_{2}(r)=(\log [\operatorname{arth}(r) / r]) / r^{2}$ is strictly increasing from $(0,1)$ onto $(1 / 3, \infty)$; $(\pi / 480, \infty)$;

(3) $f_{3}(r)=\left[\boldsymbol{\varepsilon} \operatorname{arth} r-r^{\prime 2} \mathcal{K} \operatorname{arth}(r) / 4-3 r \mathcal{K} / 4\right] / r^{5}$ is strictly increasing from $(0,1)$ onto

(4) $f_{4}(r)=(3 / 4+r / 4)\left(r-r^{\prime 2}\right.$ arth $\left.r\right) \mathcal{K}-\left(\mathcal{E}-r^{\prime 2} \mathcal{K}\right)$ arth $r$ is positive and strictly increasing in $(\sqrt{2} / 2,1)$; $(0,1 / 4)$.

(5) $f_{5}(r)=\left(3 / 4+r^{2}\right) \log [\operatorname{arth}(r) / r]-\log (2 \mathcal{K} / \pi)$ is positive and strictly increasing on

Proof. For part (1), let $h_{1}(r)=r-r^{\prime 2}$ arth $r$ and $h_{2}(r)=r^{3}$. Then $f_{1}(r)=h_{1}(r) / h_{2}(r), h_{1}(0)=$ $h_{2}(0)=0$ and

$$
\frac{h_{1}^{\prime}(r)}{h_{2}^{\prime}(r)}=\frac{2}{3} \frac{\operatorname{arth} r}{r}
$$

It is well known that the function $r \mapsto \operatorname{arth}(r) / r$ is strictly increasing from $(0,1)$ onto $(1, \infty)$. Therefore, from (2.3) and Lemma 2.1 together with l'Hôpital's rule, we know that $f_{1}(r)$ is strictly increasing in $(0,1), f_{1}\left(0^{+}\right)=2 / 3$ and $f_{1}\left(1^{-}\right)=1$. 
For part (2), clearly $f_{2}\left(1^{-}\right)=+\infty$. Let $h_{3}(r)=\log [\operatorname{arth}(r) / r]$ and $h_{4}(r)=r^{2}$, then $f_{2}(r)=h_{3}(r) / h_{4}(r), h_{3}(0)=h_{4}(0)=0$, and

$$
\frac{h_{3}^{\prime}(r)}{h_{4}^{\prime}(r)}=\frac{r-r^{\prime 2} \operatorname{arth} r}{2 r^{2} r^{\prime 2} \operatorname{arth} r}=\frac{1}{2} \frac{r-r^{\prime 2} \operatorname{arth} r}{r^{3}} \frac{r}{r^{\prime 2} \operatorname{arth} r}
$$

It follows from Lemma 2.1, Lemma 2.2(1), part (1), (2.4), and l'Hôpital's rule that $f_{2}(r)$ is strictly increasing in $(0,1)$ and $f_{2}\left(0^{+}\right)=1 / 3$.

For part (3), from Lemma 2.2(4), we clearly see that $f_{3}\left(1^{-}\right)=+\infty$. Let $h_{5}(r)=\varepsilon$ arth $r-$ $r^{\prime 2} \mathcal{K} \operatorname{arth}(r) / 4-3 r \mathcal{K}(r) / 4, h_{6}(r)=r^{5}, h_{7}(r)=\left(\mathcal{E}-r^{\prime 2} \mathcal{K}\right) /\left(4 r^{\prime 2}\right)-r \mathcal{K} \operatorname{arth}(r) / 2+3 \operatorname{arth}(r)(\varepsilon-$ $\left.r^{\prime 2} \mathcal{K}\right) /(4 r)$, and $h_{8}(r)=r^{4}$, then $f_{3}(r)=h_{5}(r) / h_{6}(r), h_{5}(0)=h_{6}(0)=h_{7}(0)=h_{8}(0)=0$,

$$
\begin{gathered}
\frac{h_{5}^{\prime}(r)}{h_{6}^{\prime}(r)}=\frac{1}{5} \frac{h_{7}(r)}{h_{8}(r)}, \\
\frac{h_{7}^{\prime}(r)}{h_{8}^{\prime}(r)}=\frac{1}{4 r^{\prime 4}} \frac{r-r^{\prime 2} \operatorname{arth} r}{r^{3}}\left[\frac{3}{4} \frac{\mathcal{\varepsilon}(r)-r^{\prime 2} \mathcal{K}(r)}{r^{2}}-\frac{1}{4} \mathcal{\varepsilon}(r)\right] .
\end{gathered}
$$

From Lemma 2.2(2) and part (1), we clearly see that $h_{7}^{\prime}(r) / h_{8}^{\prime}(r)$ is strictly increasing in $(0,1)$. Thus, the monotonicity of $f_{3}(r)$ can be obtained from (2.5) and Lemma 2.1. Moreover, making use of l'Hôpital's rule, we have $f_{3}\left(0^{+}\right)=\pi / 480$.

For part $(4)$, let $h_{9}(r)=2(1+r)-\varepsilon /(r \mathcal{K})-3\left(\varepsilon-r^{\prime 2} \mathcal{K}\right) /\left(r^{2} \mathcal{K}\right)$. Then, Lemma 2.2(3) leads to the conclusion that $h_{9}(r)$ is strictly increasing in $(0,1)$. Note that

$$
\begin{gathered}
h_{9}\left(\frac{\sqrt{2}}{2}\right)=1.013 \cdots>0, \\
f_{4}\left(\frac{\sqrt{2}}{2}\right)=0.084 \cdots>0, \\
f_{4}^{\prime}(r)=\frac{(\mathcal{K}-\varepsilon)+r \mathcal{K}(r)}{4(1+r)}+\frac{r \mathcal{K} \operatorname{arth} r}{4} h_{9}(r)>\frac{r \mathcal{K} \operatorname{arth} r}{4} h_{9}\left(\frac{\sqrt{2}}{2}\right)>0
\end{gathered}
$$

for $r \in(\sqrt{2} / 2,1)$.

Therefore, part (4) follows from (2.7) and (2.8).

For part (5), simple computations lead to

$$
\begin{gathered}
\lim _{r \rightarrow 0^{+}} f_{5}(r)=0, \\
f_{5}^{\prime}(r)=2 r \log \left(\frac{\operatorname{arth} r}{r}\right)+\left(\frac{3}{4}+r^{2}\right) \frac{r-r^{\prime 2} \operatorname{arth} r}{r r^{\prime 2} \operatorname{arth} r}-\frac{\varepsilon-r^{\prime 2} \mathcal{K}}{r r^{\prime 2} \mathcal{K}} .
\end{gathered}
$$


Making use of parts (1)-(4), one has

$$
\begin{aligned}
\frac{r^{\prime 2} \mathcal{K} \operatorname{arth} r}{r^{4}} f_{5}^{\prime}(r) & =\frac{2 r^{\prime 2} \mathcal{K} \operatorname{arth} r}{r} f_{2}(r)+\mathcal{K} f_{1}(r)-f_{3}(r) \\
& >K f_{1}(r)-f_{3}(r)>\frac{\pi}{3}-f_{3}\left(\frac{1}{4}\right)=1.040 \cdots>0
\end{aligned}
$$

for $r \in(0,1 / 4)$.

Therefore, part (5) follows from (2.9) and (2.11).

Lemma 2.4. Let

$$
g_{c}(r)=\left(\frac{3}{4}+c r\right) \log \left[\frac{\operatorname{arth}(r)}{r}\right]-\log \left(\frac{2 \mathcal{K}}{\pi}\right) \quad(c \in \mathbb{R})
$$

then the following statements are true:

(1) $g_{c}(r)>0$ for all $r \in(0,1)$ if and only if $c \in[1 / 4, \infty)$;

(2) $g_{c}(r)<0$ for all $r \in(0,1)$ if and only if $c \in(-\infty, 0]$.

Proof. Firstly, we prove that $g_{c}(r)>0$ for $c \in[1 / 4, \infty)$. Since $g_{c}(r)$ is continuous and strictly increasing with respect to $c \in \mathbb{R}$ for fixed $r \in(0,1)$, it suffices to prove that $g_{1 / 4}(r)>0$ for all $r \in(0,1)$. Note that

$$
\begin{gathered}
\lim _{r \rightarrow 0^{+}} g_{1 / 4}(r)=0, \\
g_{1 / 4}^{\prime}(r)=\frac{1}{4} \log \left(\frac{\operatorname{arth} r}{r}\right)+\left(\frac{3}{4}+\frac{1}{4} r\right) \frac{r-r^{\prime 2} \operatorname{arth} r}{r r^{\prime 2} \operatorname{arth} r}-\frac{\varepsilon-r^{\prime 2} \mathcal{K}}{r r^{\prime 2} \mathcal{K}} .
\end{gathered}
$$

We divide the proof into two cases.

Case $1(r \in(0, \sqrt{2} / 2])$. Then, making use of Lemma 2.3(1)-(3) and (2.14), we have

$$
\begin{aligned}
\frac{r^{\prime 2} \mathcal{K} \text { arth } r}{r^{3}} g_{1 / 4}^{\prime}(r) & =\frac{r^{\prime 2} \mathcal{K} \text { arth } r}{4 r} f_{2}(r)+\frac{1}{4} \mathcal{K}(r) f_{1}(r)-r f_{3}(r) \\
& >\frac{1}{4} \mathcal{K}(r) f_{1}(r)-r f_{3}(r)>\frac{\pi}{12}-\frac{\sqrt{2}}{2} f_{3}\left(\frac{\sqrt{2}}{2}\right) \\
& =0.250 \cdots>0 .
\end{aligned}
$$

Case $2(r \in(\sqrt{2} / 2,1))$. Then, making use of Lemma 2.3(4) and (2.14), we get

$$
\frac{g_{1 / 4}^{\prime}(r)}{\log [\operatorname{arth}(r) / r]}=\frac{1}{4}+\frac{f_{4}(r)}{r r^{\prime 2} \mathcal{K} \operatorname{arth} r \log [\operatorname{arth}(r) / r]}>0 .
$$

Inequalities (2.15) and (2.16) imply that $g_{1 / 4}(r)$ is strictly increasing in $(0,1)$. Therefore, $g_{1 / 4}(r)>0$ follows from (2.13) and the monotonicity of $g_{1 / 4}(r)$. 
On the other hand, inequality (1.5) leads to the conclusion that $g_{c}(r)<0$ for all $r \in$ $(0,1)$ and $c \in(-\infty, 0]$.

Next, we prove that the parameters $1 / 4$ and 0 are the best possible parameters in Lemma 2.4(1) and (2), respectively.

If $c \in(0,1 / 4)$, then $g_{c}(c)=f_{5}(c)>0$ follows from Lemma 2.3(5). Moreover, let

$$
F(r)=\frac{g_{c}(r)}{\log [\operatorname{arth}(r) / r]}=\frac{3}{4}+c r-\frac{\log (2 \mathcal{K} / \pi)}{\log [\operatorname{arth}(r) / r]},
$$

then, using l'Hôpital's rule and Lemma 2.2(4), we get

$$
\lim _{r \rightarrow 1^{+}} F(r)=c-\frac{1}{4}<0 .
$$

Inequality (2.18) implies that there exists $\delta=\delta(c)>0$ such that $F(r)<0$ for all $r \in(1-\delta, 1)$. Therefore, $g_{c}(r)<0$ for $r \in(1-\delta, 1)$ follows from $(2.17)$.

From Lemma 2.4, we clearly see that the following Theorem 2.5 holds, which give a positive answer to the open problem \#.

Theorem 2.5. The double inequality

$$
\frac{\pi}{2}\left(\frac{\operatorname{arth} r}{r}\right)^{3 / 4+\alpha^{*} r}<\mathcal{K}(r)<\frac{\pi}{2}\left(\frac{\operatorname{arth} r}{r}\right)^{3 / 4+\beta^{*} r}
$$

holds for all $r \in(0,1)$ with the best possible constants $\alpha^{*}=0$ and $\beta^{*}=1 / 4$.

\section{Acknowledgments}

This paper was supported by the Natural Science Foundation of China under Grant 11071069 and the Innovation Team Foundation of the Department of Education of Zhejiang Province under Grant T200924.

\section{References}

[1] F. Bowman, Introduction to Elliptic Functions with Applications, Dover Publications, New York, NY, USA, 1961.

[2] P. F. Byrd and M. D. Friedman, Handbook of Elliptic Integrals for Engineers and Scientists, Springer-Verlag, New York, NY, USA, 1971.

[3] G. D. Anderson, S.-L. Qiu, and M. K. Vamanamurthy, "Elliptic integral inequalities, with applications," Constructive Approximation, vol. 14, no. 2, pp. 195-207, 1998.

[4] G. D. Anderson, M. K. Vamanamurthy, and M. Vuorinen, Conformal Invariants, Inequalities, and Quasiconformal Maps, John Wiley \& Sons, New York, NY, USA, 1997.

[5] G. D. Anderson, M. K. Vamanamurthy, and M. Vuorinen, "Distortion functions for plane quasiconformal mappings," Israel Journal of Mathematics, vol. 62, no. 1, pp. 1-16, 1988.

[6] M. Vuorinen, "Singular values, Ramanujan modular equations, and Landen transformations," Studia Mathematica, vol. 121, no. 3, pp. 221-230, 1996.

[7] G. D. Anderson, S.-L. Qiu, M. K. Vamanamurthy, and M. Vuorinen, "Generalized elliptic integrals and modular equations," Pacific Journal of Mathematics, vol. 192, no. 1, pp. 1-37, 2000. 
[8] S.-L. Qiu, "Grötzsch ring and Ramanujan's modular equations," Acta Mathematica Sinica, vol. 43, no. 2, pp. 283-290, 2000 (Chinese).

[9] S.-L. Qiu, M. K. Vamanamurthy, and M. Vuorinen, "Some inequalities for the Hersch-Pfluger distortion function," Journal of Inequalities and Applications, vol. 4, no. 2, pp. 115-139, 1999.

[10] M. K. Vamanamurthy and M. Vuorinen, "Inequalities for means," Journal of Mathematical Analysis and Applications, vol. 183, no. 1, pp. 155-166, 1994.

[11] R. W. Barnard, K. Pearce, and K. C. Richards, "A monotonicity property involving ${ }_{3} F_{2}$ and comparisons of the classical approximations of elliptical arc length," SIAM Journal on Mathematical Analysis, vol. 32, no. 2, pp. 403-419, 2000.

[12] R. W. Barnard, K. Pearce, and K. C. Richards, "An inequality involving the generalized hypergeometric function and the arc length of an ellipse," SIAM Journal on Mathematical Analysis, vol. 31, no. 3, pp. 693-699, 2000.

[13] R. W. Barnard, K. Pearce, and L. Schovanec, "Inequalities for the perimeter of an ellipse," Journal of Mathematical Analysis and Applications, vol. 266, no. 2, pp. 295-306, 2001.

[14] G. D. Anderson, M. K. Vamanamurthy, and M. Vuorinen, "Functional inequalities for complete elliptic integrals and their ratios," SIAM Journal on Mathematical Analysis, vol. 21, no. 2, pp. 536-549, 1990.

[15] G. D. Anderson, M. K. Vamanamurthy, and M. Vuorinen, "Functional inequalities for hypergeometric functions and complete elliptic integrals," SIAM Journal on Mathematical Analysis, vol. 23, no. 2, pp. 512-524, 1992.

[16] H. Alzer and S.-L. Qiu, "Monotonicity theorems and inequalities for the complete elliptic integrals," Journal of Computational and Applied Mathematics, vol. 172, no. 2, pp. 289-312, 2004.

[17] Á. Baricz, "Turán type inequalities for generalized complete elliptic integrals," Mathematische Zeitschrift, vol. 256, no. 4, pp. 895-911, 2007.

[18] S. András and Á Baricz, "Bounds for complete elliptic integral of the first kind," Expositiones Mathematicae, vol. 28, no. 4, pp. 357-364, 2010. 


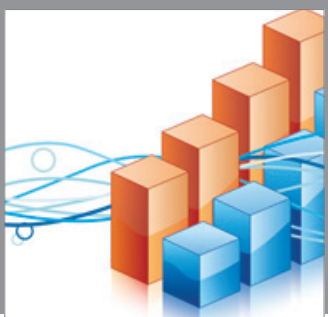

Advances in

Operations Research

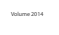

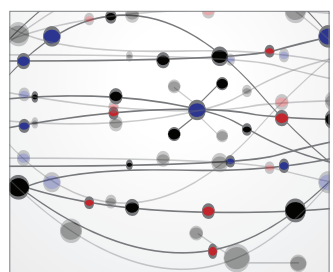

\section{The Scientific} World Journal
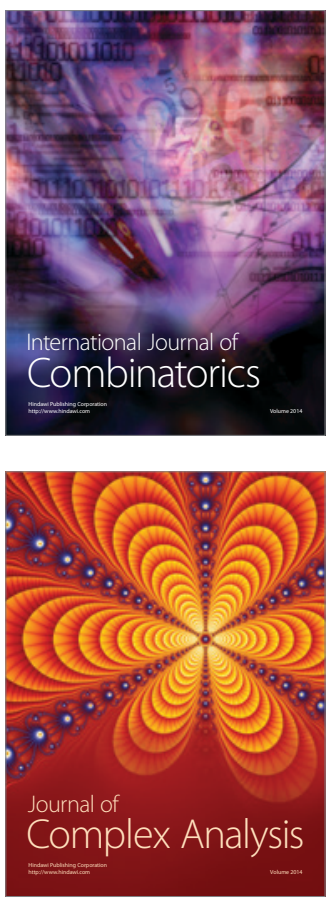

International Journal of

Mathematics and

Mathematical

Sciences
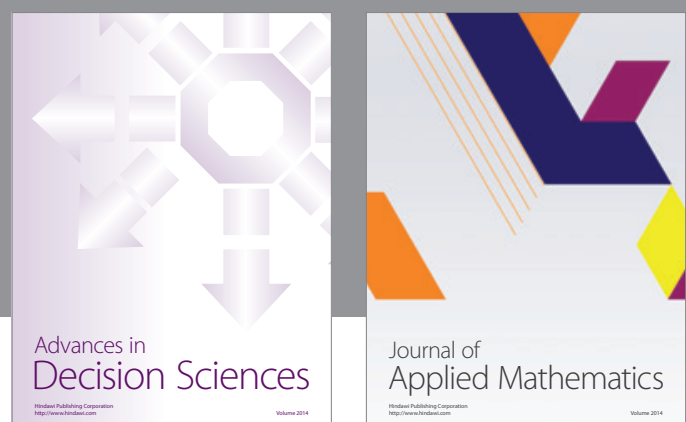

Journal of

Applied Mathematics
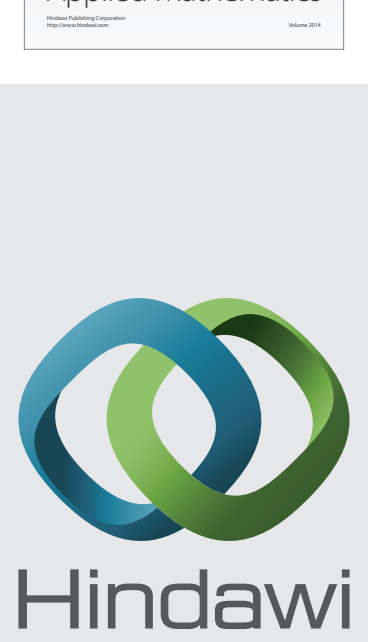

Submit your manuscripts at http://www.hindawi.com
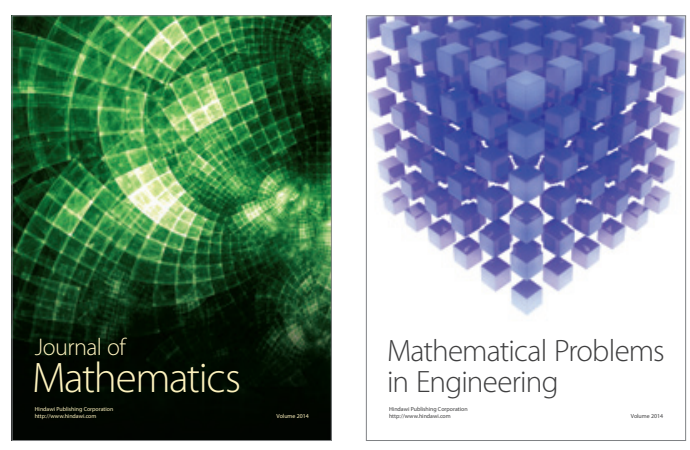

Mathematical Problems in Engineering
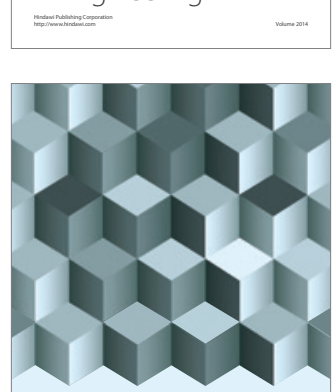

Journal of

Function Spaces
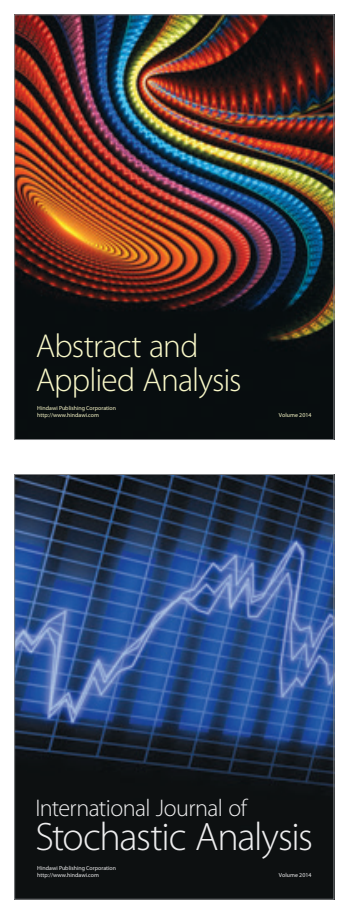

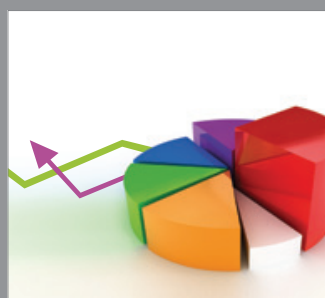

ournal of

Probability and Statistics

Promensencen
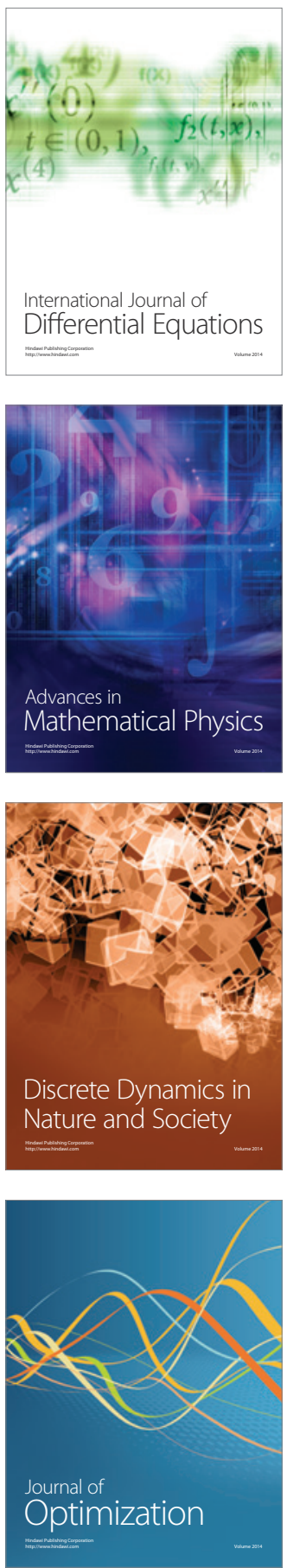